\title{
Rheumatoid Vasculitis as an Initial Presentation of Rheumatoid Arthritis
}

\author{
Sravani Lokineni, Mohamed Amr, Leela Krishna Teja Boppana, Megha Garg \\ Department of Internal Medicine, Rochester Regional Health, Rochester, NY, USA
}

Received: $14 / 04 / 2021$

Accepted: $16 / 04 / 2021$

Published: 29/04/2021

How to cite this article: Lokineni S, Amr M, Boppana LKT, Garg M. Rheumatoid vasculitis as an initial presentation of rheumatoid arthritis. EJCRIM 2021;8: doi:10.12890/2021_002561.

Conflicts of Interests: The authors declare there are no competing interests.

This article is licensed under a Commons Attribution Non-Commercial 4.0 License

\section{ABSTRACT}

Rheumatoid vasculitis is a rare, extra-articular manifestation that can be seen in long-standing rheumatoid arthritis. Here we present the case of a 51-year-old man who presented with arthralgias, skin rash, dyspnoea and generalized leg swelling and who was diagnosed with rheumatoid arthritis flare.

\section{LEARNING POINTS}

- Extra-articular manifestations like rheumatoid vasculitis and pericarditis rarely present as initial manifestations of rheumatoid arthritis.

- A high index of suspicion is required to make the diagnosis, especially in an adult who presents with multiorgan manifestations, rash, and a high titre of rheumatoid factor and anti-CCP antibody levels.

\section{KEYWORDS}

Rheumatoid vasculitis, rheumatoid arthritis, pericarditis

\section{CASE DESCRIPTION}

A 51-year-old Caucasian man with a history of tobacco use presented to the emergency department with shortness of breath and progressive pain and swelling in his upper and lower extremities for 3 weeks as well as an erythematous rash over his lower abdomen and thighs that had started 1 day previously. He reported joint pain in his hands for the previous 6 months and was not responding to NSAIDS. He also reported progressive muscle weakness for 6 months and a weight loss of 9 pounds. There was no family history of autoimmune diseases.

The patient's vital signs were normal. A physical examination showed bilateral 2+ lower extremity oedema, jugular venous distension, no murmur and clear lungs, with diffuse swelling and bilateral tenderness in the wrists, knees and ankles. Non-scaly, non-blanching, violaceous, slightly raised lesions were present on the anterior aspect of both thighs (Fig. 1A). A neurological examination showed $3 / 5$ power with no clear distal or proximal predominance in the upper limbs and 2/5 power in the hip flexors. C-reactive protein and the erythrocyte sedimentation rate were elevated at $109 \mathrm{mg} / \mathrm{l}$ (normal range: 0-10) and $74 \mathrm{~mm} / \mathrm{hr}$ (normal range: 0-20), respectively. Brain natriuretic peptide was $186 \mathrm{pg} / \mathrm{ml}$ (normal range: 0-100). Rheumatoid factor (RF) was 1,700 IU/ml (normal range: 0.1-3.9), and cyclic citrullinated peptide antibodies (CCP) were $>300 \mathrm{U} / \mathrm{ml}$ (normal: $<3$ ). A transthoracic echocardiogram showed abnormal septal motion consistent with right ventricle volume overload and a small, circumferential pericardial effusion. A nerve conduction study showed severe sensorimotor neuropathy with axonal degeneration. Cardiac magnetic resonance imaging showed a circumferentially thickened pericardium with diffuse pericardial hyperenhancement, suggesting pericardial inflammation consistent with constrictive pericarditis. A skin biopsy of the thigh lesions showed leucocytoclastic vasculitis (Fig. 1B). Given the findings of joint pain, vasculitis and constrictive pericarditis with elevated CCP and RF, the patient was diagnosed with rheumatoid arthritis flare. He was treated with pulse dose steroids for 2 days and transitioned to high-dose oral prednisone with a slow taper with an improvement in his swelling, rash and inflammatory markers. 
He was discharged to follow-up with rheumatology and started on methotrexate, sulfasalazine and eventually, rituximab infusions. Later, he was admitted for a pericardiectomy due to failure of conservative management of constrictive pericarditis.
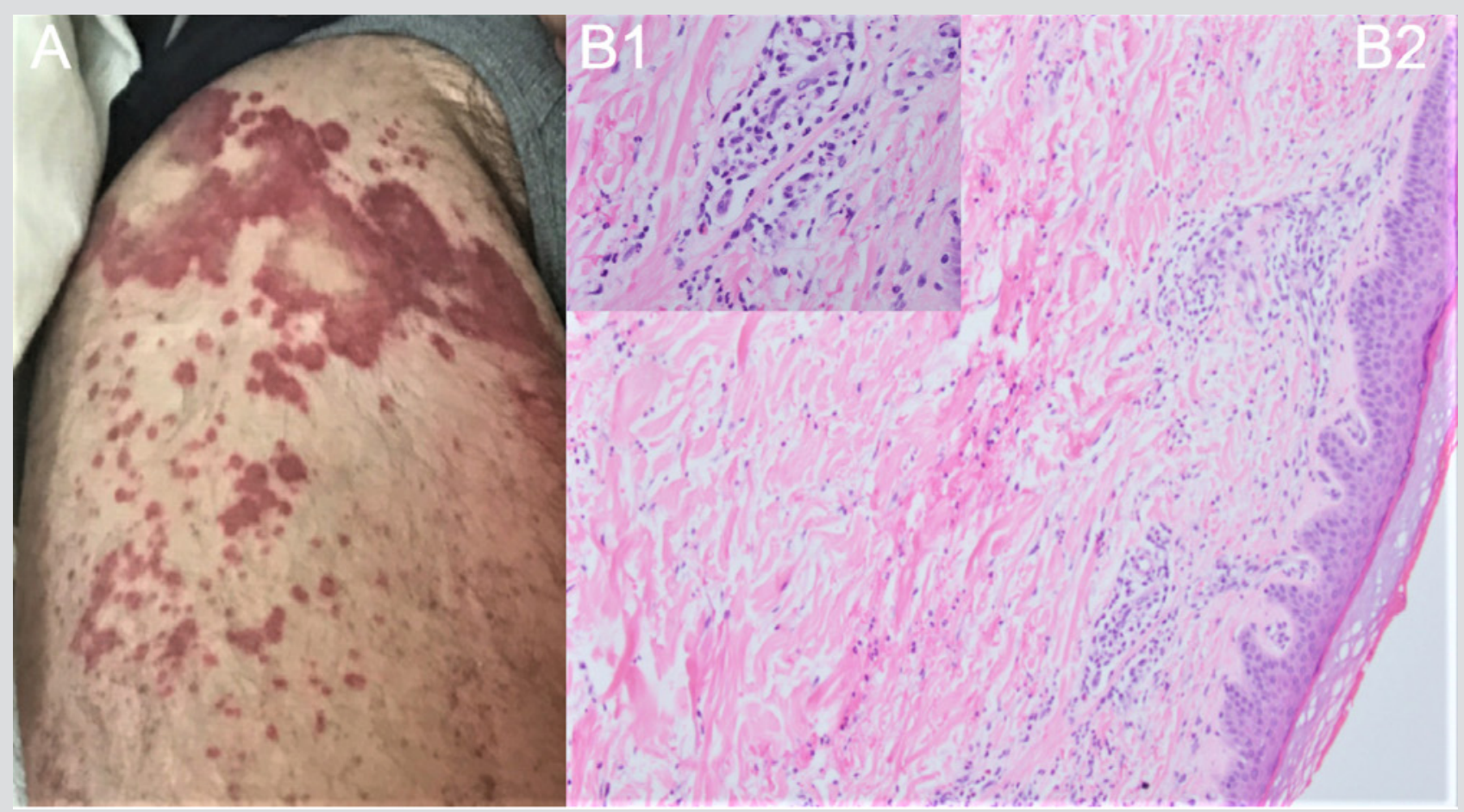

Figure 1. (A) Non-scaly, non-blanching, violaceous, slightly raised lesions present on the anterior aspect of the thigh. (B1, B2) Normal epidermis, dermal infiltrate in low power (10x), neutrophilic infiltrate around the blood vessels, and extravasated red blood cells in high power (100x)

\section{DISCUSSION}

The presentations of rheumatoid arthritis are heterogeneous and not always straightforward. Extra-articular manifestations involving the skin and the heart are rarely the initial presenting symptoms and are usually associated with increased morbidity and mortality. Rheumatoid vasculitis (RV) is a rare but potentially severe manifestation in patients with long-standing seropositive rheumatoid arthritis (RA). The mean duration [1] between the diagnosis of RA and the onset of vasculitis is 10-14 years. Rheumatoid vasculitis causes the inflammation and narrowing of small and medium sized blood vessels, typically in the skin, digits, eyes, peripheral nerves and heart. A retrospective study of 41 cases with RV from 1975 to 2017 reported that constitutional symptoms like fever and weight loss were seen in 30 (73\%) patients and extraarticular manifestations in 17 (41\%) patients, with the most common being cutaneous manifestations in 28 patients (68\%) and polyneuritis manifestations in 26 patients $(63 \%)^{[2,3]}$.

$\mathrm{RV}$ criteria include the presence of one or more of the following in a patient with RA: 1) mononeuritis multiplex or peripheral neuropathy, 2) peripheral gangrene, 3) biopsy evidence of acute necrotizing arteritis plus systemic illness (e.g., fever and weight loss), and 4) deep cutaneous ulcers or extra-articular disease if associated with typical digital infarcts or biopsy evidence of vasculitis ${ }^{[4]}$.

Laboratory work-up shows high titres of RF and anti-CCP antibody levels, which is a strong predictor for RV development. A skin biopsy can aid in diagnosing RV by showing inflammatory infiltrate and fibrinoid deposits in the blood vessel wall ${ }^{[2]}$. Management includes high-dose pulse steroids, disease-modifying anti-rheumatic drugs, and biologic agents such as rituximab ${ }^{[1,3]}$.

This case serves as a reminder that rheumatoid arthritis can sometimes present as vasculitis and/or heart failure secondary to constrictive pericarditis. These presentations are rare, so keeping a high index of suspicion is the key to making the diagnosis. 


\section{REFERENCES}

Makol A, Matteson EL, Warrington KJ. Rheumatoid vasculitis: an update. Curr Opin Rheumatol 2015;27(1):63-70.

Ntatsaki E, Mooney J, Scott DGI, Watts RA. Systemic rheumatoid vasculitis in the era of modern immunosuppressive therapy. Rheumatology 2014:53(1):145-152.

Olivé A, Riveros A, Juárez P, Morales-Ivorra I, Holgado S, Narváez J. Rheumatoid vasculitis: a study of 41 cases. Med Clin (Barc) 2020;155(3):126-129.

Scott DG, Bacon PA. Intravenous cyclophosphamide plus methylprednisolone in treatment of systemic rheumatoid vasculitis. Am J Med 1984;76(3):377-384. 